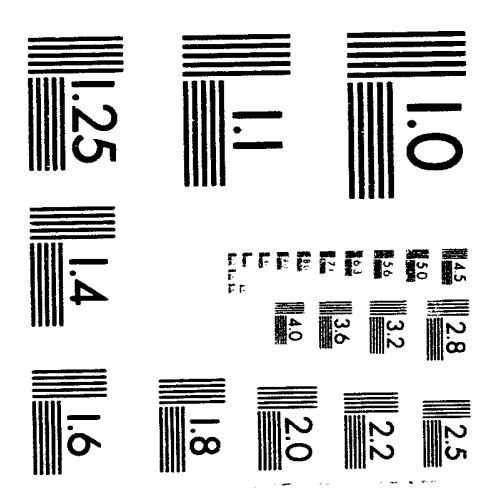



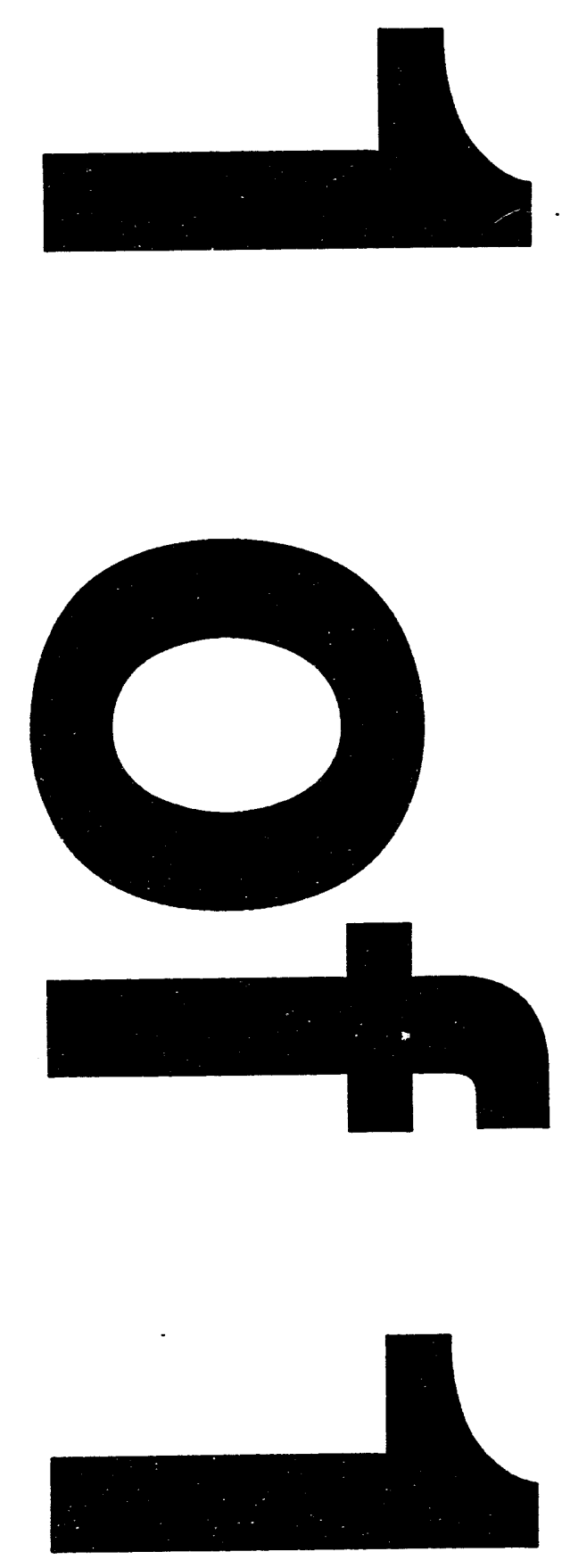
SAND93-7072

Distribution

Unlimited Release

Category UC-705

Printed February 1994

\title{
THERM3D \\ A Boundary Element Computer Program for Transient Heat Conduction Problems
}

\author{
Marc S. Ingber \\ Sandia National Laboratories \\ Albuquerque, NM 87185
}

\begin{abstract}
The computer code THERM3D implements the direct boundary element method (BEM) to solve transient heat conduction problems in arbitrary three-dimensional domains. This particular implementation of the BEM avoids performing timeconsuming domain integrations by approximating a "generalized forcing function" in the interior of the domain with the use of radial basis functions. An approximate particular solution is then constructed, and the original problem is transformed into a sequence of Laplace problems. The code is capable of handling a large variety of boundary conditions including isothermal, specified flux, convection, radiation, and combined convection and radiation conditions. The computer code is benchmarked by comparisons with analytic and finite element results.
\end{abstract}




\section{Contents}

1 Introduction 1

2 Boundary Element Formulation $\quad 2$

3 Program Description $\quad 8$

4 Program Usage $\quad 10$

5 Benchmark Demonstration Problems 14

5.1 Problem 1 ...................... 14

5.2 Problem $2 \ldots \ldots \ldots \ldots \ldots$

5.3 Problem $3 \ldots \ldots \ldots \ldots$

5.4 Problem 4 ..................... 19

$\begin{array}{llr}6 & \text { Discussion } & 20\end{array}$

$\begin{array}{lll}7 & \text { References } & 21\end{array}$ 


\section{List of Figures}

1 Local node number convention for the 6 -node triangular and 9-node quadrilateral elements. . . . . . . . . . . . . . 10

2 Temperatures at two interior points for benchmark problem 2. 18

3 Comparison of analytic, semi-analytic, and BEM results for the problem of transient heat conduction in a sphere. . . . . 18 


\section{Introduction}

The boundary element method (BEM) has become an alternative technique to domain methods such as the finite difference and finite element methods for solving partial differential equations. The method has proven to be particularly conducive for a wide class of linear elliptic boundary value problems because of the the inherent reduction in the dimensionality of the problem. In fact, in many cases, the BEM requires only the discretization of the boundary of the domain. This can be particularly advantageous in a design setting where the creation of a discretized representation of the solid model often represents the major portion of the overall effort. Unfortunately, this advantage is generally lost for nonelliptic problems. Nevertheless, the BEM has been successfully applied to a variety of parabolic and hyperbolic partial differential equations.

Several boundary element formulations have previously been developed to solve parabolic equations. The first boundary element formulation used to solve the diffusion equation was proposed by Rizzo and Shippy [1]. Rizzo and Shippy eliminated the time derivative using the Laplace transform to reduce the problem to an elliptic form and then employed a direct BEM. The inverse transform was performed numerically at a discrete set of points. However, as the number of points increased, the matrix associated with the inversion process became ill-conditioned and led to numerical instabilities [2]. Some improvements to the inversion process have recently been developed to mitigate some of the problems caused by this ill-conditioning [3].

The next genre of BEMs applied to the diffusion equation used a timedependent Green's function $[2,4,5]$. These methods, designated as total Green's function methods, retained the full dimensionality of the problem. The associated integrals possessed integrands that approached Dirac delta functions as the time step was reduced. Because of quadrature errors after a certain point, solution errors actually increased with a reduction in the time step [6]. Taigbenu and Liggett [7] developed a method which performed the time integrations analytically to avoid the problem associated with these large quadrature errors for small time steps.

The third genre of BEMs used discretization in time $[6,8,9]$ transforming 
the parabolic equation into a sequence of either Poisson or nonhomogeneous Helmholtz equations. In particular, these methods avoided the cumbersome inverse transforms required by the Laplace transform methods and eliminated the time integrations required by the total Green's function methods. However, the early BEM formulations based on discretization in time required performing domain integrations. For some BEMs, domain integrations could account for well over $50 \%$ of the total CPU effort. Several techniques have recently been developed for eliminating the domain integrals associated with boundary element methods [10-13].

The method developed in this work also uses discretization in time. The value of the dependent variable at the current time step is simply considered as an additional, nonhomogeneous forcing term. Therefore, the analysis reduces to essentially solving a sequence of Poisson problems. However, instead of performing a domain integration as typical with classical BEM algorithms for the solution of the Poisson problem, an approximate particular solution is constructed. Using a transformation of variables involving the approximate particular solution, the original problem can be transformed into solving a sequence of Laplace equations.

We discuss the boundary element formulation in Section 2. A brief discussion of the program organization of THERM3D is provided in Section 3 followed by a users manual in Section 4. Several benchmark application problems are presented in Section 5 to show the capabilities and performance of THERM3D.

\section{Boundary Element Formulation}

We consider the heat conduction equation with constant conductivity $k$, density $\rho$, and specific heat $c_{p}$ in a three dimensional domain $\Omega$ bounded by the surface $\Gamma$. The governing equation is given by

$$
\rho c_{p} \frac{\partial u}{\partial t}+g=k \nabla^{2} u
$$

where $u$ is the temperature and $g$ represents internal heat generation. The boundary conditions for this problem can be any combination of the following 
types:

$$
\begin{aligned}
\text { Prescribed temperature : } & u=\bar{u} \\
\text { Prescribed flux : } & q=-\bar{q} \\
\text { Convection: } & q=-h\left(u-u_{\infty}\right) \\
\text { Radiation : } & q=-\sigma c\left(u^{4}-u_{\infty}^{4}\right) \\
\text { Convection, radiation: } & q=-h\left(u-u_{\infty}\right)-\sigma \epsilon\left(u^{4}-u_{\infty}^{4}\right)
\end{aligned}
$$

where $q$ is the heat flux defined by $q=k \partial u / \partial n, n$ is the outward unit normal vector, $h$ is the heat transfer coefficient, $\sigma$ is the Stefan-Boltzmann constant, $\epsilon$ is the emissivity, and $u_{\infty}$ is the ambient temperature. The initial condition is given by

$$
u(\mathbf{x}, 0)=f(\mathbf{x})
$$

We employ the method of discretization in time, whereby the spatial variables are discretized using the boundary element method and time is discretized using the finite difference method. In particular, we use the generalized trapezoidal method $(\theta$-method) to approximate the time derivative. That is, we set

$$
\frac{1}{\alpha} \frac{u^{n}-u^{n-1}}{\Delta t}=\theta\left(\nabla^{2} u^{n}-g^{n} / k\right)+(1-0)\left(\nabla^{2} u^{n-1}-g^{n-1} / k\right)
$$

where the diffusivity, $\alpha$, is defined by $\alpha=k / \rho c_{p}, \Delta t$ is the time step, $u^{n}=$ $u(\mathbf{x}, n \Delta t)$, and $g^{n}=g(\mathbf{x}, n \Delta t)$. Defining

$$
v^{n}=u^{n}-\frac{\theta-1}{\theta} u^{n-1}
$$

we can rearrange the above equation as

$$
\nabla^{2} v^{n}=\frac{v^{n}}{\theta \alpha \Delta t}-\frac{1}{\theta^{2} \alpha \Delta t} u^{n-1}+\frac{(1-\theta)}{\theta k} g^{n-1}+g^{n} / k
$$

In the current BEM approach, we consider the right-hand side of (6) to be a "generalized" forcing function. Following standard formulation procedures 
for the BEM (see, e.g., [14]), $v^{n}$ may be represented in terms of a boundary integral as follows:

$$
\begin{array}{r}
\eta(\mathbf{x}) v^{n}(\mathbf{x})=\int_{\Gamma}\left[G^{\prime}(\mathbf{x}, \xi) v^{n}(\xi)-G(\mathbf{x}, \xi) v^{n^{\prime}}(\xi)\right] d \Gamma(\xi) \\
+\int_{\Omega}\left[\frac{v^{n}(\xi)}{\theta \alpha \Delta t}-\frac{1}{\theta^{2} \alpha \Delta t} u^{n-1}(\xi)+\frac{(1-\theta)}{\theta k} g^{n-1}(\xi)+g^{n}(\xi) / k\right] G(\mathbf{x}, \xi) d \Omega(\xi)
\end{array}
$$

where $G(\mathbf{x}, \xi)$ is the Green's function given by $G(\mathbf{x}, \xi)=\frac{1}{|\mathbf{x}-\xi|}$, the prime denotes the derivative in the direction of the outward normal to the boundary at the point $\xi$, and the coefficient $\eta(\mathbf{x})$ can be determined from the integral

$$
\eta(\mathbf{x})=\int_{\Gamma} G^{\prime}(\mathbf{x}, \xi) d \Gamma(\xi)
$$

The major difficulty with this approach is that we have introduced a domain integral which contains the unknown function $v^{n}(\xi)$. Although $v^{n}$ can be discretized in the interior using nodal basis functions, the evaluation of the domain integral is usually very time consuming. In order to eliminate the domain integral, we approximate

$$
f(\mathbf{r})=\frac{v^{n}}{\theta \alpha \Delta t}-\frac{1}{\theta^{2} \alpha \Delta t} u^{n-1}+\frac{(1-\theta)}{\theta k} g^{n-1}+g^{n} / k \approx \sum_{i=1}^{M} \alpha_{i}^{n} \psi_{i}(\mathbf{r})
$$

The functions $\psi_{i}$ have the form

$$
\psi_{i}(\mathbf{r})=\psi\left(\left|\mathbf{r}-\mathbf{r}_{\mathbf{i}}\right| / \beta_{i}\right)
$$

where $\mathbf{r}_{\mathbf{i}}$ and $\beta_{i}$ are constants. The $\psi_{i}$ 's are called radial basis functions.

There is a wide variety of possibilities for the functional form of the radial basis functions and the parameters $\alpha_{i}, \beta_{i}$, and $\mathbf{r}_{\mathbf{i}}$. For implementation in THERM3D, we choose

$$
\psi_{i}(\mathbf{r})=\left(1+r^{2}\right)^{-1 / 2}
$$

where $r=\left|\mathbf{r}-\mathbf{r}_{\mathbf{i}}\right| / \beta_{\mathbf{i}}$. The $\mathbf{r}_{\mathbf{i}}$ 's are chosen to be fairly evenly distributed points within the interior of the domain, $\Omega$, and the $\beta_{i}$ 's are chosen to be the same order of magnitude as the distance of the closest neighbors to $\mathbf{r}_{\mathbf{i}}$. 
These choices for the parameters lead to a good interior approximation for $f(\mathbf{r})$ which can be determined from a well-conditioned set of linear equations for the $\alpha_{i}$ 's [13]. A good reference on the theory of radial basis functions which discusses in some detail the selection of the appropriate parameters is provided by Powell [15].

Collocating (8) at each of the $\mathrm{M}$ interior points $\mathbf{r}_{\mathbf{i}}$ yields a set of equations which can be written in matrix form as

$$
\left[\psi_{m i}\right]\left\{\alpha_{i}^{n}\right\}=\frac{\tilde{v}_{m}^{n}}{\alpha \theta \Delta t}-\frac{u_{m}^{n-1}}{\alpha \theta^{2} \Delta t}+g_{m}^{n} / k+\frac{(1-\theta)}{\theta k}-g_{m}^{n-1}
$$

where $\tilde{v}_{m}^{n}, u_{m}^{n-1}$, and $g_{m}^{n}$ represent the values of $v, u$ and $g$, respectively, at the $\mathrm{m}^{\text {th }}$ interior node $\mathbf{r}_{m}$ at the time step indicated by the superscript. The tilde is used to distinguish $v$ in the interior from $v$ on the boundary in the subsequent discussion. This equation can be rearranged into the following form

$$
\left\{\tilde{v}_{m}^{n}\right\}=\alpha \theta \Delta t\left[\psi_{m i}\right]\left\{\alpha_{i}^{n}\right\}+\frac{1}{\theta}\left\{u_{m}^{n-1}\right\}-\frac{\alpha \theta \Delta t}{k}\left[\left\{g_{m}^{n}\right\}+\frac{(1-\theta)}{\theta}\left\{g_{m}^{n-1}\right\}\right]
$$

Associated with the radial basis function, $\psi(r)$, is a particular solution $\phi(r)$ such that

$$
\nabla^{2} \phi=\frac{1}{r^{2}} \frac{d}{d r}\left(r^{2} \frac{d \phi}{d r}\right)=\psi(r)
$$

The particular solution is given by

$$
\phi(r)=\frac{1}{2}\left[\left(1+r^{2}\right)^{1 / 2}+\frac{\ln \left(r+(1+r)^{1 / 2}\right)}{r}\right]
$$

Hence, an approximate particular solution of $(6), v_{p}^{n}(r)$, at the $n$th time step is given by

$$
v_{p}^{n}(r)=\sum_{i=1}^{M} \alpha_{i}^{n} \phi_{i}(r)
$$

We now consider the change of variables

$$
v_{g}^{n}=v^{n}-v_{p}^{n}
$$


Then the governing equation for $v_{g}^{n}$ is given by

$$
\nabla^{2} v_{g}^{n}=0 \quad \mathrm{x} \in \Omega
$$

The boundary conditions associated with the transformed problem can be determined from (2). The boundary integral equation associated with the transformed problem no longer contains a domain integral term as in (7). That is,

$$
\eta(\mathbf{x}) v_{g}^{n}(\mathbf{x})=\int_{\Gamma}\left[G^{\prime}(\mathbf{x}, \xi) v_{g}^{n}(\xi)-G(\mathbf{x}, \xi) v_{g}^{n^{\prime}}(\xi)\right] d \Gamma(\xi)
$$

Collocating (18) at each of the boundary element nodes yields a set of $n$ linear equations, which can be written in matrix form as

$$
\left[A_{i j}\right]\left\{v_{g_{j}}^{n}\right\}=\left[B_{i j}\right]\left\{q_{g_{j}}^{n}\right\}
$$

where $\left\{v_{g_{j}}^{n}\right\}$ represents the value of the general solution, $v_{g}$, at the $n$th time step at the $j$ th boundary element node and $\left\{q_{g_{j}}^{n}\right\}$ represents the corresponding flux (normal derivative). The boundary conditions for the transformed problem are related to the particular solution which in turn can be related to the coefficients $\alpha_{\imath}$ of the interior approximation by

$$
\left\{v_{p_{j}}^{n}\right\}=\left[\Phi_{j i}\right]\left\{\alpha_{i}^{n}\right\} \quad\left\{q_{p}^{n}\right\}=\left[\Phi_{j i}^{\prime}\right]\left\{\alpha_{i}^{n}\right\}
$$

where $\Phi_{j i}$ represents the value of $\phi_{i}$ at the $j$ th boundary element node and $\Phi_{j i}^{\prime}$ represents the value of the normal derivative of $\phi_{i}$ at the $j$ th boundary element node. Inserting (16) and (20) into (19) yields

$$
\left[A_{i j}\right]\left\{v_{j}^{n}\right\}-\left[A_{i j}\right]\left[\Phi_{j k}\right]\left\{\alpha_{k}^{n}\right\}=\left[B_{i j}\right]\left\{q_{j}^{n}\right\}-\left[B_{i j}\right]\left[\Phi_{j k}^{\prime}\right]\left\{\alpha_{k}^{n}\right\}
$$

Since the interior approximation given by (8) is indeterminate, the $\alpha_{i}^{n} \mathrm{~s}$ have been introduced into (21) as additional unknowns. Therefore, it is necessary to introduce additional equations in order to close the algebraic system. This is accomplished by collocating (18) at the $M$ interior points $\mathbf{r}_{\mathbf{i}}$ associated with the radial basis functions. The resulting equations can be written as

$$
\left\{\tilde{v}_{g_{i}}^{n}\right\}+\left[C_{i j}\right]\left\{v_{g_{j}}^{n}\right\}=\left[\Gamma_{i j}\right]\left\{q_{g_{j}}^{n}\right\}
$$


where $\tilde{v}_{g_{i}}^{n}$ represents the general solution, $v_{g}$, at the interior point $\mathbf{r}_{\mathbf{i}}$. In the interior, the transformed variable is given by

$$
\tilde{v}_{g_{i}}^{n}=\tilde{v}_{i}^{n}-\tilde{v}_{p_{i}}^{n}
$$

where $\tilde{v}_{i}^{n}$ and $\tilde{v}_{p_{i}}^{n}$ represent the values of $v$ and associated particular solution $v_{p}$, respectively, at $\mathbf{r}_{\mathbf{i}}$ at the $n$th time step. Again, $\tilde{v}_{p_{i}}^{n}$ can be related to the $\alpha_{i}^{n}$ s through the equation

$$
\left[\tilde{\Phi}_{j i}\right]\left\{\alpha_{i}^{n}\right\}=\left\{\tilde{v}_{p_{j}}^{n}\right\}
$$

where $\tilde{\Phi}_{j i}$ represents the value of $\phi_{i}$ at $\mathbf{r}_{\mathbf{j}}$. Inserting (12), (23), and (24) into (22) yields

$$
\begin{aligned}
\alpha \theta \Delta t\left[\Psi_{m i}\right]\left\{\alpha_{i}^{n}\right\}-\left[\tilde{\Phi}_{m i}\right]\left\{\alpha_{i}^{n}\right\}+\frac{1}{\theta}\left\{u_{m}^{n-1}\right\}-\frac{\alpha \theta \Delta t}{k}\left[\left\{g_{m}^{n}\right\}+\frac{1-\theta}{\theta}\left\{g_{m}^{n-1}\right\}\right] \\
+\left[C_{m j}\right]\left\{v_{j}^{n}\right\}-\left[C_{m j}\right]\left[\Phi_{j k}\right]\left\{\alpha_{k}^{n}\right\}=\left[D_{m j}\right]\left\{q_{j}^{n}\right\}-\left[D_{m j}\right]\left[\Phi_{j k}^{\prime}\right]\left\{\alpha_{k}^{n}\right\}
\end{aligned}
$$

The system of equations given by (21) and (25) can now be solved for $\left\{v_{j}^{n}\right\}$ and $\left\{\alpha_{k}^{n}\right\}$ by standard techniques to trace the time history of the boundary element solution. As a practical matter, all matrices can be determined outside the time loop. Further, an LU decomposition can also be performed outside the time loop. Hence, the only operations performed within the time loop are matrix-vector multiplications and back-substitution.

The actual assembly of the iterative system of equations is somewhat involved. The prescribed temperature, flux, and convection conditions pose no special problem. However, the radiation condition is non-linear and requires some attention. Although it is possible to use a Newton method to impose the radiation conditions, this would then require that the coefficient matrix be factored at each iteration within each time step. It is thus more efficient to use Picard's method of direct substitution to impose the radiation boundary conditions even though the convergence rate is slower compared to Newton's method. Hence, we treat the radiation boundary condition similarly to the condition of specified flux. That is, we specify

$$
q_{i}^{k, n}=-\sigma \epsilon\left[\left(u_{i}^{k-1, n}\right)^{4}-u_{\infty}^{4}\right]
$$


where the first superscript indicates the iteration number, the second superscript indicates the time step, and the subscript indicates the boundary element node number. The value for $u_{i}^{0, n}$ comes from the solution at the previous time step. Convergence is obtained when the $L_{2}$-norm for the vector $\left\{q_{i}^{k, n}\right\}$ is less than a prescribed tolerance. It is sometimes necessary to underrelax the iteration in order to achieve convergence.

\section{Program Description}

The program THERM3D and the 23 associated subroutines are listed in the Appendix. The time stepping is performed in the main program. A brief description of the prominent subroutines is given below.

GEOM: This subroutine reads the problem data from the file "therm3d.dat". The subroutine also calculates the average values of the outward-normal vectors at the boundary element nodes.

MATRICES: This subroutine collects and assembles the various matrices necessary to form the linear system of equations. These matrices include AMAT $\left(\left[A_{i j}\right]\right)$ and BMAT $\left(\left[B_{i j}\right]\right)$ from $(19)$, CMAT $\left(\left[C_{i j}\right]\right)$ and DMAT $\left(\left[D_{i j}\right]\right)$ from (22), PHIMAT $\left(\left[\Phi_{i j}\right)\right]$ and PHIPMAT $\left(\left[\Phi_{i j}^{\prime}\right)\right]$ from (20), PHITMAT $\left(\left[\tilde{\Phi}_{i j}\right)\right]$ from (24) and PSIMAT $\left(\left[\psi_{i j}\right)\right]$ from (11). All these matrices are time independent, and hence, are evaluated once outside the time loop.

INT4,RTINT,STINT,RQINT,SQINT: The subroutine INT4 is the driving subroutine to perform the integrations along the boundary necessary to determine $\left[A_{i j}\right],\left[B_{i j}\right],\left[C_{i j}\right]$, and $\left[D_{i j}\right]$ once the collocation point has been chosen. For each element, the subroutine determines the coordinates associated with the nodes of the element making use of the element connectivity. Depending on the element type and whether the collocation point resides in the element (in which case the integral is singular), the subroutine calls either RTINT for the regular integration over a triangular element, STINT for the 
singular integration over a triangular element, RQINT for the regular integration over a quadrilateral element, or SQINT for the singular integration over a quadrilateral element.

RHS: This subroutine performs the matrix-vector multiplications necessary to form the right hand side of the global linear equations. The subroutine is called within every iteration within every time step.

LUDCMP,LUBKSB: These subroutines are taken from the book Numerical Recipes [16]. LUDCMP performs an LU decomposition of the coefficient matrix GMAT outside the time loop. LUBKSB performs back substitution within the time loop to determine the solution at the boundary element and internal nodes.

CHKCONV: This subroutine checks the convergence of the fluxes on the radiation boundaries. If the convergence criterion is met then the temperatures in the interior are updated and the program goes to tile next time step. If the convergence criterion is not met then the iteration counter is incremented and the iteration is continued.

CALU: This subroutine outputs the temperatures at the boundary element and interior nodes determined from the boundary element solution at prescribed time intervals.

CALPS: This subroutine is called from MATRICES and calculates the values of the particular solutions $\phi_{i}(r)$ and their normal derivatives for collocation points on the boundary to form $\left[\Phi_{i j}\right]$ and $\left[\Phi_{i j}^{\prime}\right]$ as required by $(21)$ and (25). (See Section 2.)

CALPI: This subroutine is called from MATRICES and calculates the values of the particular solutions $\phi_{i}(r)$ and the radial basis functions $\psi_{i}(r)$ for collocation points in the interior to form $\left[\tilde{\Phi}_{i j}\right]$ and $\left[\Psi_{i j}\right]$ as required by $(25)$. (See Section 2.)

CALG: This is a user defined subroutine to calculate the values of the function $g$ as a function of position and time which represents the internal heat 
generation. THERM3D comes with a default subroutine CALG which simply sets $g \equiv 0$.

\section{Program Usage}

Problem data are read from the input file "therm3d.dat". Output is written to the output file "therm3d.out". All READ statements are unformatted except for the 3 title cards which have format A. The standard FORTRAN convention is followed whereby input parameter names starting with the letters $i$ through $n$ represent integer quantities. All other parameters represent real quantities except for the the three title variables. The element library consists of the 6-node triangular isoparametric element and the 9node quadrilateral isoparametric element. These elements along with the convention of the local node numbering are shown in Figure 1. It does not matter which corner node is labeled locally as node 1 , as long as the remaining nodes follow the pattern snown in the figure going around the element in the counterclockwise direction.
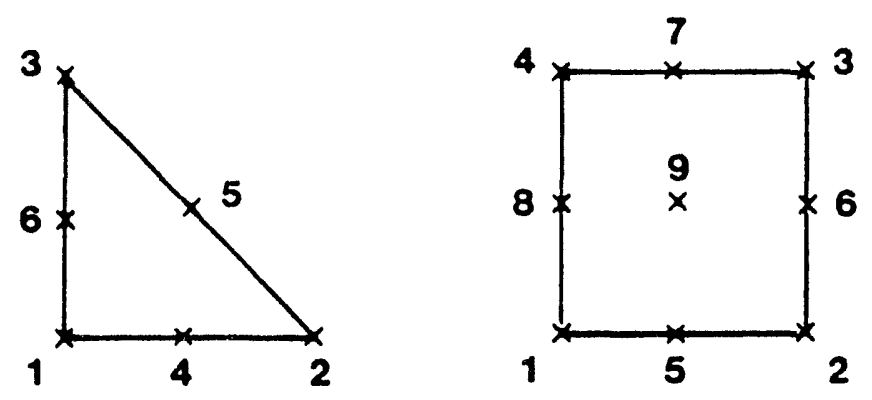

Figure 1: Local node number convention for the 6-node triangular and 9-node quadrilateral elements.

For problems with internal heat generation, the user is required to supply the subroutine "calg" which calculates the values of the function $g$ in Eq. 1 as 
a function of position and time. THERM3D comes with a default subroutine "calg" which simply returns $g \equiv 0.0$. The parameter list for the subroutine is

$$
x i(i), y i(i), z i(i), t, d t, g, g m 1
$$

The parameters $x i(i), y i(i), z i(i), t$, and $d t$ are input to the subroutine and represent the coordinates of the interior points, time, and the time step. The parameters $g$ and $g m 1$ are output from the subroutine and represent $g(x i(i), y i(i), x i(i), t)$ and $g(x i(i), y i(i), z i(i), t-d t)$, that is, the values of the function $g$ at the interior point at the current time step and the previous time step, respectively.

The structure of the input file "therm3d.dat" along with the definitions of the input parameters is given below.

\section{CARD PACKET 1:}

Cards 1 to 3 - title1,title2,title3

Card 4 - nodes, ne,nint, bfact

Card 5 - ndt, dt, theta

Card 6 - nout1, nout2

Card 7 - cond,alpha,tinf,hcoef,irflag

If irflag $=1$ then

Card 8 - sigma,epsi,imax,tol,omega

title1 - first title card

title2 - second title card

title3 - third title card

nodes - total number of boundary element nodes

ne - total number of boundary elements

nint - total number of interior points (see Note 1)

bfact - the factor $\beta$ used in the interior approximation

by the radial basis functions (see Note 1)

ndt - number of time steps

$\mathrm{dt}$ - time step 
theta - parameter $\theta$ used in the generalized trapezoidal rule (see Note 2)

nout 1 - interior temperatures are printed every "nout1" time steps

nout2 - surface temperatures are printed every "nout2" time steps

cond - thermal conductivity $(k)$

alpha - thermal diffusivity $\left(\alpha=k / \rho c_{p}\right)$

tinf - ambient far-field temperature $\left(T_{\infty}\right)$

hcoef - heat transfer coefficient $(h)$

irflag - radiation flag

irflag $=0 \Rightarrow$ there are no radiation boundaries

irflag $=1 \Rightarrow$ there are radiation boundaries

sigma - Stefan-Boltzmann constant $(\sigma)$

epsi - emissivity between the surface and the exterior environment $(\epsilon)$

imax - maximum number of iterations allowed for convergence

tol - tolerance for convergence (see Note 3)

omega - underrelaxation parameter (see Note 4)

\section{CARD PACKET 2:}

Cards 1 to nodes - i, x,y,z,nbdy,uold,par 1

$\mathrm{i}$ - global boundary node number

$x$ - $x$-coordinate

$y-y$-coordinate

$z$ - z-coordinate

nbdy - If the temperature is prescribed at the node, nbdy $=1$

If the heat flux is prescribed at the node, nbdy $=2$

If the node lies on a convection boundary, nbdy $=3$

If the node lies on a radiation boundary, nbdy $=4$

If the node lies on a convection, radiation boundary, nbdy $=5$

uold - initial temperature at the boundary element node

par1 - If $n b d y=2, \operatorname{par} 1$ is the prescribed flux

If $n b d y \neq 2, \operatorname{par} 1$ is a dummy variable 


\section{CARD PACKET 3:}

Cards 1 to ne - i,iel,n1,n2,n3,n4,n5,n6,n7,n8,n9

i - boundary element number

iel - element type

If the element is a 9 -node quadrilateral element, iel $=4$

If the element is a 6 -node triangular element, iel $=3$

$\mathrm{nj}, \mathrm{j}=1,9$ - the global node number associated with local node $\mathrm{j}$ within the $i$ th element

\section{CARD PACKET 4:}

Cards 1 to nint - i, xi,yi,zi,tinit

$i$ - interior point number

$\mathrm{xi}$ - $\mathrm{x}$-coordinate of the $i$ th interior point

$y i-y$-coordinate of the $i$ th interior point

$\mathrm{zi}$ - z-coordinate of the $i$ th interior point

tinit - initial temperature of the $i$ th interior point

Note 1: As discussed in Section 2, THERM3D requires a number of interior points in order to perform an interior approximation of the particular solution. In general, these points should be evenly spaced (although this is not a requirement). The input variable "bfact" is used to define the radial basis function (see Eq. 9) and, in general, is a measure of the region of influence of the basis function about the interior point. Large values for bfact smear the influence of the radial basis function over a larger volume about the interior point. Small values for bfact limit the influence of the basis function to be localized very close to the interior point. The variable bfact should be chosen to be the order of magnitude of the distance between adjacent interior points. 
Note 2: The variable "theta" is used in the generalized trapezoidal rule to discretize time (see Eq. 4). Currently, the program can accept values of $\theta$ such that $0<\theta \leq 1$. In particular, forward differencing $(\theta=0)$ is not allowed. Common values for $\theta$ are 1 (backwards differencing) and $1 / 2$ (Crank-Nicholson).

Note 3: The variable "tol" is the tolerance required for convergence for radiation problems. After each iteration, THERM3D calculates the temperature at each node on the radiation boundary. The $L_{2}$ norm of the difference between the vector comprised of the current values of temperature along the radiation boundary and the vector comprised of the values of temperature from the previous iteration is then calculated. This norm is then checked against the tolerance to determine whether to go to the next time step or continue the iteration.

Note 4: The variable "omega" $(\omega)$ is the underrelaxation parameter. When updating the flux on the radiation boundary, $(1-\omega)$ times the current value is added to $\omega$ times the old value. Allowable values for $\omega$ are $0 \leq \omega<1$. If $\omega=0$ then then there is no underrelaxation. In the third benchmark problem discussed in the next section, no underrelaxation was required to achieve convergence. In the fourth benchmark problem, underrelaxation was required. For that problem, an omega of 0.4 yielded the fastest convergence.

\section{Benchmark Demonstration Problems}

\subsection{Problem 1}

The governing equation for the first problem is given by

$$
\frac{\partial u}{\partial t}=\frac{\partial^{2} u}{\partial x^{2}}+\frac{\partial^{2} u}{\partial y^{2}}+\frac{\partial^{2} u}{\partial z^{2}}+g(x, y, z, t)
$$

to be solved in the domain $0 \leq x, y, z \leq 1$. The forcing function is given by

$$
g(x, y, z, t)=\sin x \sin y \sin z(3 \sin t+\cos t)
$$


The initial and boundary conditions are appropriate to the solution

$$
u=\sin x \sin y \sin z \sin t
$$

The dependent variable $u$ is prescribed on the face $x=1$ and the flux $q$ is prescribed on all other faces. This problem is of interest since the boundary conditions and the forcing function are time dependent.

We considered a course and a fine mesh for this example. The course mesh contained 150 surface nodes, 24 boundary elements, and 27 interior points. The fine mesh contained 486 surface nodes, 96 boundary elements, and 343 interior points. Results at the interior point $x=0.75, y=0.75$, $z=0.75$ for the coarse and fine meshes using a time step of 0.1 are displayed in Table 1. The results determined using the coarse mesh were generally within $4 \%$ of the exact values, and the results determined using the fine mesh were generally within $0.4 \%$. For this example, the results generated using backward differencing $(\theta=1)$ were slightly more accurate than results generated using the Crank-Nicholson method $(\theta=1 / 2)$ for the coarse mesh while the opposite was true for the fine mesl. Results at the interior point $x=$ $0.5, y=0.625, z=0.875$ generated using the fine mesh but two different time steps are shown in Table 2. The convergence of the method is demonstrated in the tables.

\subsection{Problem 2}

This problem considers a cubic region $0 \leq x, y, z \leq 0.5$ which is initially at $1^{\circ} \mathrm{C}$. The sides with $x=0, y=0$, and $z=0$ are insulated. For time $t>0$, the remaining three sides are placed in a convective environment with far field temperature equal to zero. That is, convection boundary conditions are specified on three sides of the cube. The thermal diffusivity and heat transfer coefficients are given by $\alpha=1.0$ and $h=1.0$. The same course and fine meshes used with example problem 1 are again used for this problem. A time step of 0.025 is chosen for this problem. Results at two typical inierior points are shown in Figure 2. It can be seen that the fine grid yields 
Table 1: Results for the interior point $x=0.75, y=0.75, z=0.75$ generated using the course mesh and fine mesh with $\Delta t=0.1$.

\begin{tabular}{|c|c|c|c|c|c|}
\hline \hline & \multicolumn{2}{|c|}{$\theta=1$} & \multicolumn{2}{c|}{$\theta=0.5$} & \\
\hline Time & Coarse Mesh & Fine Mesh & Coarse Mesh & Fine Mesh & Analytic \\
\hline 0.4 & 0.1189 & 0.1229 & 0.1173 & 0.1231 & 0.1233 \\
0.8 & 0.2193 & 0.2263 & 0.2174 & 0.2270 & 0.2272 \\
1.2 & 0.2856 & 0.2941 & 0.2838 & 0.2952 & 0.2952 \\
1.6 & 0.3069 & 0.3154 & 0.3056 & 0.3168 & 0.3166 \\
2.0 & 0.2797 & 0.2869 & 0.2792 & 0.2884 & 0.2880 \\
2.4 & 0.2085 & 0.2131 & 0.2087 & 0.2144 & 0.2139 \\
2.8 & 0.1043 & 0.1057 & 0.1054 & 0.1067 & 0.1061 \\
3.2 & -0.0163 & -0.0184 & -0.0147 & -0.0179 & -0.0185 \\
3.6 & -0.1344 & -0.1396 & -0.1324 & -0.1397 & -0.1402 \\
4.0 & -0.2312 & -0.2388 & -0.2292 & -0.2394 & -0.2397 \\
\hline \hline
\end{tabular}

Table 2: Results for the interior point $x=0.5, y=0.625, z=0.875$ generated using the fine mesh and two different time steps.

\begin{tabular}{|c|c|c|c|c|c|}
\hline \hline & \multicolumn{2}{|c|}{$\theta=1$} & \multicolumn{2}{c|}{$\theta=0.5$} & \\
\hline Time & $\Delta t=0.2$ & $\Delta t=0.1$ & $\Delta t=0.2$ & $\Delta t=0.1$ & Analytic \\
\hline 0.4 & 0.0832 & 0.0835 & 0.0838 & 0.0837 & 0.0838 \\
0.8 & 0.1528 & 0.1538 & 0.1549 & 0.1546 & 0.1545 \\
1.2 & 0.1982 & 0.1999 & 0.2017 & 0.2011 & 0.2007 \\
1.6 & 0.2124 & 0.2144 & 0.2167 & 0.2160 & 0.2152 \\
2.0 & 0.1929 & 0.1951 & 0.1975 & 0.1969 & 0.1958 \\
2.4 & 0.1430 & 0.1449 & 0.1472 & 0.1465 & 0.1454 \\
2.8 & 0.0705 & 0.0719 & 0.0736 & 0.0731 & 0.0721 \\
3.2 & -0.0131 & -0.0124 & -0.0116 & -0.0118 & -0.0126 \\
3.6 & -0.0947 & -0.0948 & -0.0950 & -0.0949 & -0.0953 \\
4.0 & -0.1613 & -0.1622 & -0.1633 & -0.1630 & -0.1629 \\
\hline \hline
\end{tabular}


more accurate results again demonstrating the convergence of the boundary element formulation.

We performed some timings on an HP 715 workstation for this problem to determine how much of the CPU effort was being expended inside and outside of the time loop. For the coarse mesh, 17.7 CPU seconds were required outside the time loop. Recall that all of the matrices are evaluated outside of the time loop and the $\mathrm{LU}$ factorization is performed outside the time loop. For every time step, $0.26 \mathrm{CPU}$ seconds were required within the time loop to perform the matrix-vector multiplications and to perform the back substitution. For the fine mesh, $339 \mathrm{CPU}$ seconds were required outside the time loop, and 3.8 CPU seconds were required inside the time loop per time step. From these timings, it is evident that most of the computational overhead resides outside the time loop.

\subsection{Problem 3}

This problem considers a sphere initially at $298 K$ which is placed in a convective and/or radiative environment with the ambient farfield temperature given by $T_{\infty}=3000 \mathrm{~K}$. The remaining physical constants are given by $k=2.0 \mathrm{~W} / \mathrm{m} \cdot K, \sigma \epsilon=5.67 e-8 \mathrm{~W} / \mathrm{m}^{2} \cdot K^{4}, h=2000 \mathrm{~W} / \mathrm{m} \cdot K$, and $\alpha=1.0 \mathrm{~m}^{2} / \mathrm{s}$. This problem was chosen so that the results could be compared with the results of a semi-analytical method developed by Walker [17].

The boundary element grid contained 48 boundary elements and 56 interior points. Three cases were considered, namely, pure convection, pure radiation, and combined radiation and convection. A time step of $\Delta t=0.0001$ was chosen for the boundary element analysis for all three cases. The results for the surface temperatures are shown in Figure 3. Note that the convection results are analytic while the radiation and convection/radiation results were calculated by Walker using her semi-analytical method. Walker compared the semi-analytic results to finite element results generated by the commercial code ABAQUS and found the agreement with the finite element code to be good. The agreement between the analytical results and the numerical results generated by THERM3D is excellent. No internal temperatures were 


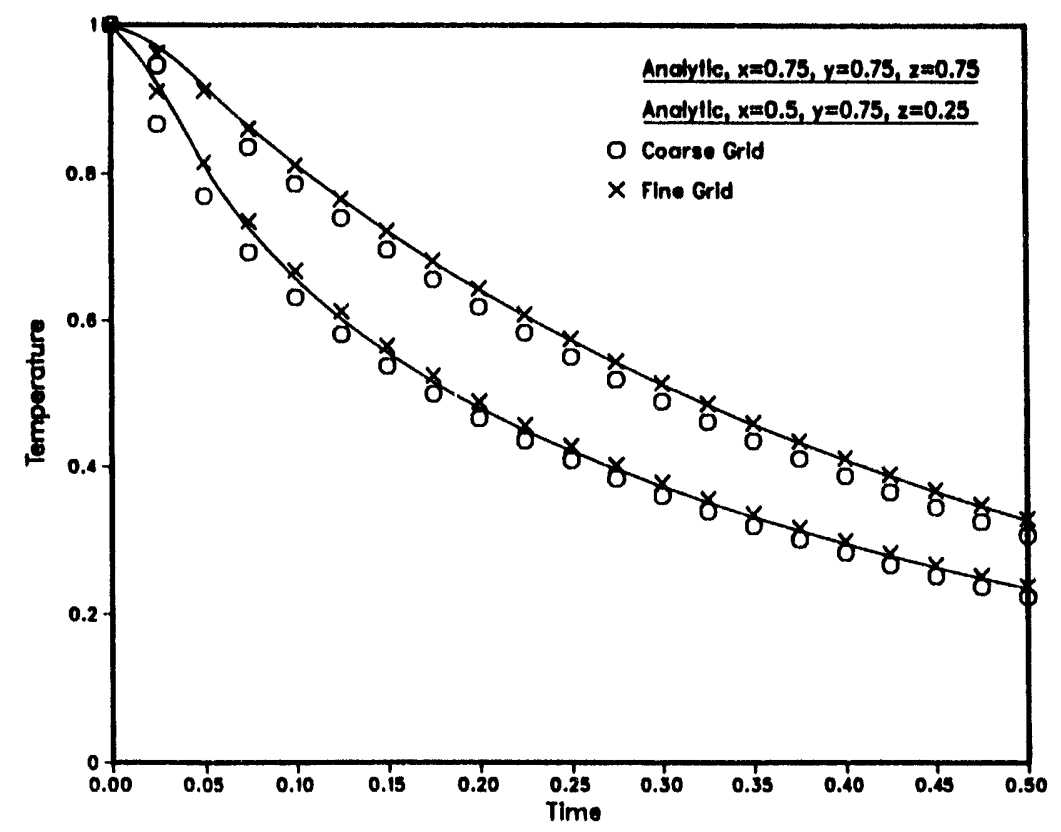

Figure 2: Temperatures at two interior points for benchmark problem 2.

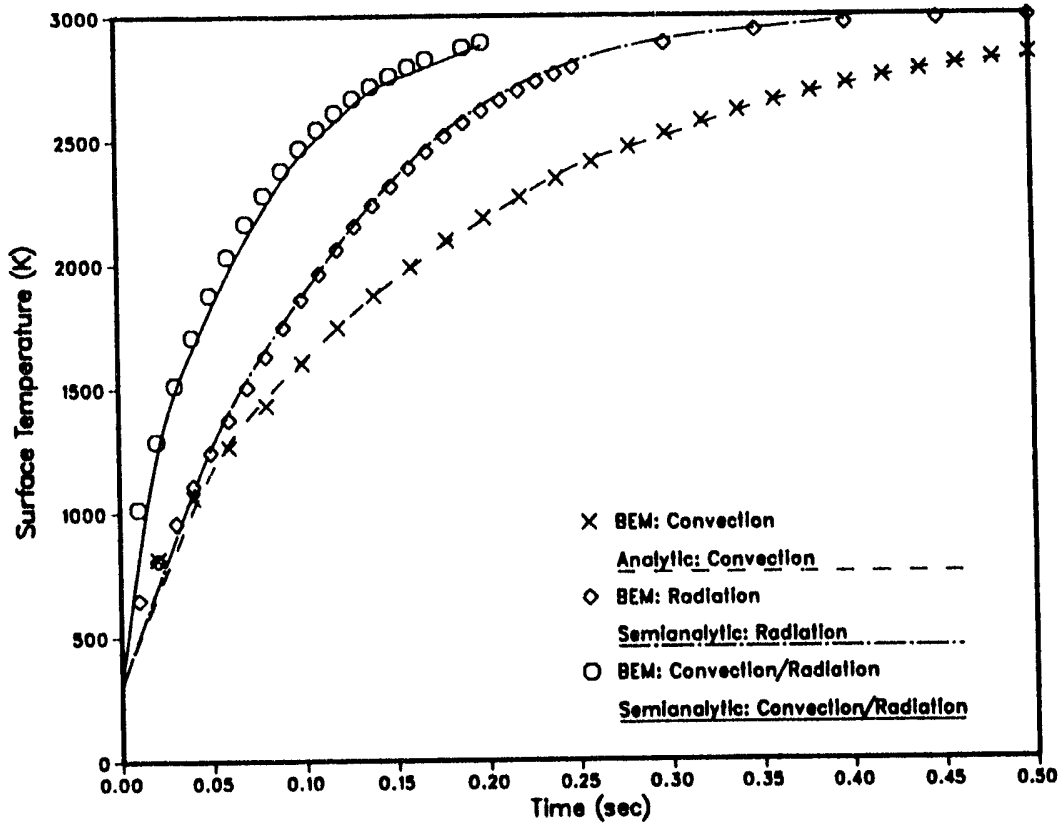

Figure 3: Comparison of analytıc, semi-analytic, and BEM results for the problem of transient heat conduction in a sphere. 
reported by Walker. For this problem, no underrelaxation was required by the BEM for convergence.

\subsection{Problem 4}

This problem considers a cube initially at $298 K$ with isothermal bottom and insulated side walls which is place in a radiative environment with the ambient farfield temperature given by $T_{\infty}=3000 \mathrm{~K}$. The remaining physical constants are given by $k=44.2686 \mathrm{~W} / \mathrm{m} \cdot K, \sigma \epsilon=5.67 e-8 \mathrm{~W} / \mathrm{m}^{2} \cdot K^{4}$, and $\alpha=1.221 e-5 \mathrm{~m}^{2} / \mathrm{s}$. This problem was chosen so that the results could be benchmarked against the finite element code COYOTE [18] and the commercial finite difference code P/THERMAL [19]. A one-dimensional finite element method (1D FEM) code was also written to analyze this problem.

Two boundary element meshes were considered for THERM3D. The coarse boundary element mesh contained 54 boundary elements and 125 interior nodes and the fine boundary element mesh contained 96 boundary elements and 343 interior nodes. Similarly, two finite element meshes were considered for COYOTE. The coarse finite element mesh contained 125 uniform finite elements and the fine finite element mesh contained 1738 uniform finite elements. Only one grid containing 196 nodal points was considered for P/THERMAL. The coarse boundary element mesh, the coarse finite element mesh, and the finite difference grid were similar in that they were constructed from identical sets of points. A time step of 1 second was chosen for THERM3D, COYOTE, and P/THERMAL. The discretization for the 1D FEM code contained 50 2-node linear elements. Further, a time step of 0.1 seconds was chosen for the 1D FEM code.

Temperatures calculated using the various codes are shown in Table 3 for the center of the cube and in Table 4 for the radiation surface of the cube. Since this benchmark problem is one-dimensional, it can be effectively analyzed using the 1D FEM code, and the discretization for the 1D FEM code is by far the finest. Therefore, we presume that the 1D FEM results are the most accurate. It is seen in the Tables 3 and 4 , that the results generated using the fine grids are closer to the 1D FEM results than the results generated using the coarse grids. Also, in general, the finite element results generated 
by COYOTE are closer to the $1 \mathrm{D}$ FEM results than the BEM results. The results generated by $\mathrm{P} / \mathrm{THERMAL}$ are comparable in accuracy to the results generated by COYOTE. The CPU times for THERM3D were 288 and 1306 seconds for the coarse and fine grids, respectively, on a SPARC2 workstation. The CPU times for COYOTE were 298 and 736 seconds for the coarse and fine grids, respectively, also on a SPARC2 workstation.

Table 3: Temperature at the center of the cube.

\begin{tabular}{|c|c|c|c|c|c|c|}
\hline \hline Time & P/THERMAL & CFE & FFE & CBE & FBE & FEM 1D \\
\hline 15 & 306.3 & 289.6 & 300.3 & 312.7 & 309.7 & 303.7 \\
30 & 366.4 & 339.3 & 358.5 & 394.6 & 382.2 & 367.5 \\
60 & 593.1 & 582.8 & 594.4 & 640.4 & 623.2 & 601.4 \\
90 & 821.0 & 820.7 & 825.7 & 866.2 & 849.9 & 830.1 \\
120 & 1004.1 & 1008.7 & 1009.7 & 1043.2 & 1029.3 & 1012.3 \\
\hline \hline
\end{tabular}

Table 4: Temperature on the top surface of the cube.

\begin{tabular}{|c|c|c|c|c|c|c|}
\hline \hline Time & P/THERMAL & CFE & FFE & CBE & FBE & FEM 1D \\
\hline 15 & 1665.3 & 1732.0 & 1753.1 & 1926.9 & 1859.0 & 1775.5 \\
30 & 2142.2 & 2146.6 & 2150.6 & 2117.0 & 2187.7 & 2159.6 \\
60 & 2461.3 & 2455.8 & 2455.2 & 2472.6 & 2464.0 & 2457.9 \\
90 & 2584.2 & 2580.5 & 2579.8 & 2587.9 & 2583.8 & 2581.1 \\
120 & 2651.5 & 2649.3 & 2648.6 & 2653.8 & 2651.3 & 2649.3 \\
\hline \hline
\end{tabular}

\section{Discussion}

The computer program THERM3D is an implementation of the direct boundary element method to discretize space and the generalized trapezoidal rule to discretize time to solve transient heat conduction problems. An approximate particular solution is generated in the interior of the domain which is used with a transformation of variables to eliminate the time-consuming volume integral associated with the boundary integral equation. In this way, the boundary element formulation essentially reduces to solving a sequence of Laplace problems. The advantages of this approach is that much of the 
computation is performed outside of the time loop. The only computation performed within the time loop is matrix-vector multiplication and backsubstitution.

The program THERM3D has been successfully tested by comparisons with analytic and numerical results in four benchmark problems. THERM3D required more CPU time compared to the FEM code COYOTE. However, it is possible that for problems with complicated geometries or problems involving moving boundaries that THERM3D might still prove to be advantageous over domain methods because a complete interior discretization is not required for THERM3D which could simplify the modeling effort.

\section{References}

1. Rizzo, F. J. and Shippy, D. J., "A Method of Solution for Certain Problems of Transient Heat Conduction," AIAA J., 8, 2004-2009, 1970.

2. Liggett, J. A. and Liu, P. L. F., "Unsteady Flow in Confined Aquifers: A Comparison of Two Boundary Integral Methods," Water Resour. Res., 15, 861-866, 1979.

3. Moridis, G. J., "Alternative Formulations of the Laplace Transform Boundary Element (LTBE) Numerical Method for the Solution of DiffusionType Equations," in Boundary Element Technology VII, C. A. Brebbia and M. S. Ingber, Eds., Computational Mechanics Publications, Southampton, 1992.

4. Wrobel, L. C. and Brebbia, C. A., "The Boundary Element Method for Steady-State and Transient Heat Conduction," in Numerical Methods in Thermal Problems, R. W. Lewis and and K. Morgan, Eds., Pineridge Press, Swansea, Wales, 1979.

5. Curran, D., Cross, M., and Lewis, B. A., "A Preliminary Analysis of Boundary Element Methods Applied to Parabolic Partial Differential Equations," in New Developments in Boundary Element Methods, C. A. Brebbia, Ed., CML Publications, Southampton, 1980. 
6. Curran, D., Cross, M., and Lewis, B. A., "Solution of Parabolic Differential Equations by the Boundary Element Method Using Discretization in Time," Appl. Math. Modelling, 4, 398-400, 1980.

7. Taigbenu, A. E. and Liggett, J. A., "Boundary Element Calculations for the Diffusion Equation," J. Eng. Mech., 111(3), 311-328, 1985.

8. Wrobel, L. C. and Brebbia, C. A., "Boundary Elements for Non-Linear Heat Conduction Problems," Comm. Appl. Num. Meth., 4, 617-622, 1988.

9. Ingber, M. S. and Phan-Thien, N., "A Boundary Element Approach for Parabolic Equations Using a Class of Particular Solutions," Appl. Math. Modelling, 16, 124-132, 1992.

10. Nardini, D. and Brebbia, C. A., "A New Approach to Free Vibration Analysis Using Boundary Elements" in Boundary Elements IV, C. A. Brebbia, Ed., Springer-Verlag, Berlin, 1982.

11. Herry, D. P., Jr. and Banerjee, P. K., "A New Boundary Element Formulation for Two- and Three-dimensional Thermoelasticity Using Particular Integrals," Int. J. Num. Meth. Engrg., 26, 2061-2078, 1988.

12. Brebbia, C. A., "On the Treatment of Domain Integrals in Boundary Elements," in Boundary Element Techniques: Applications in Engineering, C. A. Brebbia and N. G. Zamani, eds., Computational Mechanics Publications, Southampton, 1989.

13. Zheng, R., Coleman, C. J., and Phan-Thien, N., "A Boundary Element Approach for Non-homogeneous Potential Problems," Comp. Mech., 7(4), 279-288, 1991.

14. Brebbia, C. A., Telles, J. C. F., and Wrobel, L. C., Boundary Element Techniques, Springer-Verlag, Berlin, 1984.

15. Powell, M. J. D., "Radial Basis Functions for Multivariate Interpolation," in Algorithms for Approximation, J. C. Mason and M. G. Cox, Eds., Clarendon Press, Oxford, 1987. 
16. Press, W. H., Flannery, B. P., Teukolsky, S. A., and Vetterling, W. T., Numerical Recipes, Cambridge University Press, Cambridge, 1986.

17. Walker, L. A., "Temperature-Time History of a Particle in a Radiative/Convective Environment," Masters Thesis, University of New Mexico, 1992.

18. Gartling, D. K., "COYOTE - A Finite Element Computer Program for Nonlinear Heat Conduction Problems," SAND77-1332, Sandia National Laboratories, Albuquerque, New Mexico, 1982.

19. "P/THERMAL User Manual, PDA Engineering, Patran Division, 2975 Redhill Ave., Costa Mesa, California, 1991. 
Distribution:

UC-705

MSI:1513

Copies to:

4

University of New Mexico

Mechanical Engineering Department

Albuquerque, New Mexico 87131

Attn: M. S. Ingber

$1 \mathrm{~ms} 08411500$ D. J. McCloskey

$1 \mathrm{~ms} 08361501$ C. W. Peterson

$1 \mathrm{~ms} 08271502$ P. J. Hommert

$1 \mathrm{~ms} 08271511$ D. K. Gartling

$1 \mathrm{~ms} 08271511$ C. E. Hickox

$1 \mathrm{~ms} 08271511$ M. J. Martinez

$1 \mathrm{~ms} 08271511$ J. S. Rottler

$1 \mathrm{~ms} 08351513$ R. D. Skocypec

$1 \mathrm{~ms} 08351513$ R. E. Hogan

$1 \mathrm{~ms} 08351513$ V. J. Romero

$1 \mathrm{~ms} 08351513$ Day File

$1 \mathrm{~ms} 08331552$ C. E. Hailey

$1 \mathrm{~ms} 04431561$ H. S. Morgan, route to 1562

5 ms 08997141 Technical Library

$1 \mathrm{~ms} 06197151$ Technical Publications

$10 \mathrm{~ms} 0100$ 7613-2 Document Processing for DOE/OSTI

1 ms 9018 8523-2 Central Technical Files 


$$
\longrightarrow
$$

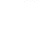
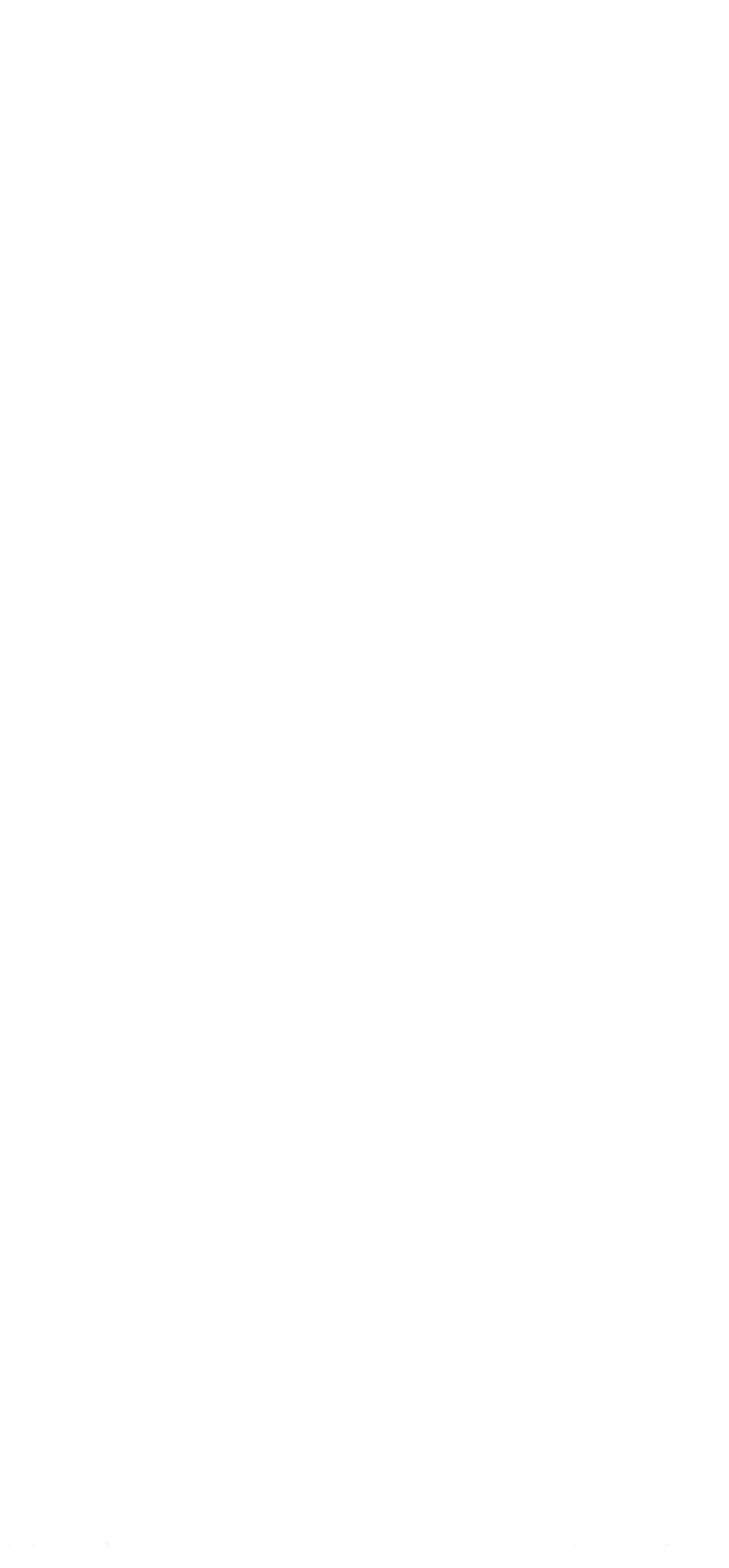